\title{
Predicting Urban Medical Services Demand in China: An Improved Grey Markov Chain Model by Taylor Approximation
}

\author{
Jinli Duan ${ }^{1,2}$, Feng Jiao ${ }^{3}$, Qishan Zhang ${ }^{2, *}$ and Zhibin Lin ${ }^{4}$ \\ 1 College of Pharmacy, Fujian University of Traditional Chinese Medicine, No. 1 Qiuyang Road, \\ Fuzhou 350122, Fujian, China; ywjoy@126.com \\ 2 School of Economics and Management, Fuzhou University, No. 2 Xueyuan Road, \\ Fuzhou 350108, Fujian, China \\ 3 Newcastle University Business School, Newcastle University, 5 Barrack Road, \\ Newcastle upon Tyne NE1 4SE, UK; f.jiao@newcastle.ac.uk \\ 4 Newcastle Business School, Northumbria University, Newcastle upon Tyne NE1 8ST, UK; \\ Zhibin.lin@northumbria.ac.uk \\ * Correspondence: zhangqs@fzu.edu.cn; Tel.: +86-136-9688-6113
}

Received: 20 June 2017; Accepted: 5 August 2017; Published: 6 August 2017

\begin{abstract}
The sharp increase of the aging population has raised the pressure on the current limited medical resources in China. To better allocate resources, a more accurate prediction on medical service demand is very urgently needed. This study aims to improve the prediction on medical services demand in China. To achieve this aim, the study combines Taylor Approximation into the Grey Markov Chain model, and develops a new model named Taylor-Markov Chain GM $(1,1)$ (T-MCGM $(1,1))$. The new model has been tested by adopting the historical data, which includes the medical service on treatment of diabetes, heart disease, and cerebrovascular disease from 1997 to 2015 in China. The model provides a predication on medical service demand of these three types of disease up to 2022. The results reveal an enormous growth of urban medical service demand in the future. The findings provide practical implications for the Health Administrative Department to allocate medical resources, and help hospitals to manage investments on medical facilities.
\end{abstract}

Keywords: medical services demand; Grey Markov chain; Taylor Approximation; prediction

\section{Introduction}

The population aged 65 and over has increased almost twice as fast as the younger population in the past two decades [1]. This sharp increase of the aging population has brought enormous pressure to the current medical services system. Moreover, improved living standards, advanced medical science and technology, and higher medical service expectations have further stimulated the demand for medical services [2]. Allocating the limited health care resources has been more challenging than ever before for policy-makers and managers. Developing an accurate and flexible forecasting system for predicting medical services demand thus becomes imperative [3,4].

To predict individual medical services demand, an early study by Grossman [5] applied Becker's equilibrium analysis framework by integrating a utility function, family production function, income constraint function, and time constraint function. Later studies by Hupert et al. [6], McCarthy et al. [7], Lowthian et al. [8], and Hagihara et al. [9] developed different models based on multiple regression analysis [6-15]. In addition, there are other forecasting models for predicting medical services demand. For example, the auto regressive moving average model combined with a neural network was established to predict the incidence of scarlet fever, which showed that the forecasting accuracy of the 
combination model is superior to the simple auto regressive moving average model [16]. A model based on the service goal and trend of the vertebral method was proposed to predict regional medical services demand [17]. The work points method is used to predict annual public health service demand considering gender and age [18]. There are also studies that applied system dynamics simulation model to predict care services demand $[19,20]$.

Among the recent studies, a Markov Chain (MC) model has been widely adopted. For example, Teresa (2012) and Akita (2016) applied the MC model to calculate the transition probability of patients in treatment [21,22]. Ujjwal (2010) presented a Grey-Markov model to forecast energy consumption in India [23]. Chen (2011) presented a Grey Markov model to forecast financial crises for an enterprise [24]. Lin et al. (2013) used a Grey-Markov forecasting model to analyze Taiwan's IC assembly industry [25]. Kordnoori et al. (2014) applied a Grey Markov model to analyze global ICT development [26]. Edem et al. (2016) proposed a modified Grey-Markov model to predict fire accidents [27]. Peng (2009) proposed a MC model considering the medical service project cost present value to predict medical services demand [28]. Huang used the continuous time homogeneous Markov process to analysis the change of the elderly population health status, to predict long-term care and nursing demand for the over 65-year-old elderly population [29]. Hu (2015) applied the MC model to estimate the elderly health state transition probability, and combined this with the result of population prediction to forecast the demand of elderly and nursing services in different years and different disability states [30]. Argiento et al. (2016) attempted to improve the Bayesian framework by applying the MC model, and developed the patients' demand evolution for the further prediction [31].

The GM $(1,1)$ prediction model was created by Deng (1989), who is the founder of Grey system theory [32]. Many studies in prediction are based on small datasets [33]. Compared with general statistic models, the models based on Grey system theory such as GM $(1,1)$ only need limited data for building and predicting, which could also provide a certain degree of fitting effect to evaluate the reliability $[34,35]$. Chen $(2012)$ used the Grey GM $(1,1)$ model to predict demand of doctors and beds in Tianjin in 2013-2018 [36]. Xiang (2015) used a multi-factors Grey prediction model for health technical personnel demand forecasting. The prediction accuracy was shown to be better than that of the auto regressive model [37]. Bao (2015) applied GM $(1,1)$ model to predict the disease burden of the aged population in China [38]. Wang $(2014)$ used the GM $(1,1)$ model to predict ischemic heart disease hospitalizations in Shenzhen, China [39].

There are three major limitations in applying these methods. First, the traditional regression analysis requires a large database to guarantee prediction accuracy. Second, applying the MC model requires the calculation of disease transition probability. Disease transaction features vary across different disease categories and patients. The probability matrix of disease transaction is different from one patient to another. Third, the traditional Grey GM $(1,1)$ model is hardly able to provide accurate predication regarding random vibration and high noise. Medical service demand is highly influenced by disease spectrum, living environment, and national health policy. Historic data of medical service demand is limited.

This study aims to address the above limitations in the current medical demand prediction literature by developing a new model which is based on the traditional GM $(1,1)$ model and combines the MC model and Taylor Approximation algorithm. The traditional GM $(1,1)$ model is used to predict the medical services demand. Then the MC model improved by the Taylor Approximation algorithm is implied to estimate the transferring state of the residual sequence in the GM $(1,1)$ model to improve the prediction accuracy. The remainder of the paper is structured as follows: Section 2 describes the procedure of the improving Grey Markov Chain Model by Taylor Approximation. Empirical results for the three types of diseases, including diabetes, heart disease, and cerebrovascular disease are presented in Section 3. Section 4 provides the final comments and conclusions. 


\section{Materials and Methods}

The improved T-MCGM $(1,1)$ model is made up of three methods, including GM $(1,1)$, Markov Chain, and Taylor Approximation. The purpose of GM $(1,1)$ is to predict the medical service demand of specific diseases by adopting historic data. That of Markov Chain is to revise the absolute error in GM $(1,1)$ by estimating the transferring state of residual sequence. These two methods combined with Taylor Approximation can effectively search the optimal upper and lower bound parameters for transferring the state of the residual sequence.

\subsection{The Traditional GM $(1,1)$ Model}

The traditional Grey GM $(1,1)$ model is developed as the following procedure:

If we assume the original data series is $X^{(0)}=\left\{x^{(0)}(1), x^{(0)}(2), \ldots, x^{(0)}(n)\right\}$, and $x^{(0)}(i)>0$, $i=1,2, \ldots, n$. We can accumulate this data series and achieve a new data series, which is $X^{(1)}=$ $\left\{x^{(1)}(1), x^{(1)}(2), \ldots, x^{(1)}(n)\right\}$, and $x^{(1)}(j)=\sum_{i=1}^{j} x^{(0)}(i), j=1,2, \ldots, n$.

Moreover, the next mean generation sequence is:

$$
Z^{1}(n)=\left\{z^{1}(2), z^{1}(3), \ldots, z^{1}(n)\right\}, \text { and } z^{1}(k)=\frac{1}{2}\left[x^{1}(k-1)+x^{1}(k)\right], k=1,2, \ldots, n .
$$

Based on the above the data series, it can develop the whitenization differential equation in the GM $(1,1)$ model as the following:

$$
\frac{d x^{(1)}}{d t}+a x^{(1)}=b
$$

In addition, the difference equation in GM $(1,1)$ is:

$$
x^{0}(j)+a z^{1}(j)=b
$$

In Equations (1) and (2), $a$ is the developing coefficient, and $b$ is the grey action. If assuming

$$
\begin{aligned}
\hat{a}=[a, b]^{T} \text { as parameter list, and } Y=\left[\begin{array}{c}
x^{0}(2) \\
x^{0}(3) \\
\ldots \\
x^{0}(n)
\end{array}\right] B=\left[\begin{array}{cc}
-z^{1}(2) & 1 \\
-z^{1}(3) & 1 \\
\ldots & \ldots \\
-z^{1}(n) & 1
\end{array}\right] \text {, the parameter list can satisfy: } \\
\widehat{a}=(a, b)^{T}=\left(B^{T} B\right)^{-1} B^{T} Y
\end{aligned}
$$

Therefore, it can develop Grey Prediction equation, which is:

$$
\begin{gathered}
\hat{x}^{(1)}(i+1)=\left[x^{0}(1)-\frac{b}{a}\right] e^{i a}+\frac{b}{a} \\
x^{(0)}(i+1)=x^{1}(i+1)-x^{1}(i)=\left[x^{(0)}(1)-\frac{b}{a}\right]\left(1-e^{a}\right) e^{-a i}
\end{gathered}
$$

\subsection{Grey Markov Chain Prediction Model}

Prediction of medical service is highly influenced by random influences, such as a disease's morbidity and seasonal factors. Thus, the traditional GM $(1,1)$ model has difficulty in predicting accurate medical service demand with original data series. In order to improve the current prediction of medical services demand, the following section applies the Markov Chain model to improve the GM $(1,1)$ model, which is able to predict the transferring of the residual sequence in the GM $(1,1)$ model. The improved model can resolve the randomness and volatility of services demand, and have a more accurate prediction on medical services demands. 


\subsubsection{The Partition of Transferring}

In practice, assuming the actual medical services demands are known, the series of absolute error is obtained between the actual demands and prediction, and it is a non-stationary random series in the Markov chain. In practice, medical service demands are different because of different types of diseases. Thus, this random series can be divided into different statuses, with $r$ denoting one status. Therefore, it can achieve a state transition matrix, which is. In this matrix, each state represents an equidistant margin of error. Each prediction error can be located into the corresponding status. We use $S_{i j}$ to indicate state in residual:

$$
S_{i j} \in\left(L_{i j}, U_{i j}\right), j=1,2, \ldots, r
$$

In addition, and there are the low bound and upper bound of state in residual, which are calculated as:

$$
\begin{gathered}
L_{i j}=\min e(i)+\frac{j-1}{r}[\max e(i)-\min e(i)] \\
U_{i j}=\operatorname{mine}(i)+\frac{j}{r}[\max e(i)-\min e(i)]
\end{gathered}
$$

\subsubsection{The Establishment of the State Transition Matrix}

If we assume that $p_{i j}^{(m)}$ is the probability of the residual transferring from state $i$ to state $j$ in step $m . M_{i j}^{(m)}$ is the sample size of the residual transferring from state $i$ to state $j$ in step $m . M_{i}$ is the sample size of the residual state $i$.

Therefore, the Markov state transaction matrix consisted of $p_{i j}^{(m)}$ can be presented as:

$$
P^{(m)}=\left(\begin{array}{cccc}
p_{11}^{(m)} & p_{12}^{(m)} & \ldots & p_{1 m}^{(m)} \\
p_{21}^{(m)} & p_{22}^{(m)} & \ldots & p_{2 m}^{(m)} \\
\ldots & \ldots & \ldots & \ldots \\
p_{r 1}^{(m)} & p_{r 2}^{(m)} & \ldots & p_{r r}^{(m)}
\end{array}\right)
$$

\subsubsection{Prediction of the Grey Markov Chain Model}

If we assume $a_{i}(t)$ is the probability of state in period, it can get that $\left\{a_{i}(t), i=1,2, \ldots, r\right\}$ is the state transaction vector in the period. $a_{i}(0)$ will denote the initial probability vector of the state transaction, and it can make use of the initial probability vector and the state transaction matrix, to calculate the probability vector of the predicting objects in any period at any state. This is the model of applying MC to GM $(1,1)$, and the model is presented as follows:

$$
\begin{gathered}
\widetilde{x}^{(0)}(t+1)=\hat{x}^{(0)}(t+1)+\sum_{i}^{r} a_{i}(t) \otimes v_{i} \\
\text { And } a_{i}(t)=\left[a_{1}(t), a_{2}(t), \ldots, a_{r}(t)\right]=a_{i}(t-1) p^{(1)}
\end{gathered}
$$

In Equation (10), $\otimes v_{i}$ is the grey number exists in a certain residual interval. It can be presented as $\otimes v_{i}=\left(L_{i}, U_{i}\right)$, and $L_{i}$ and $U_{i}$ are the lower bound and upper bound of the residual interval respectively [40].

\subsection{The Development of Grey Markov Chain Model with Taylor Approximation}

The new prediction model is based on the traditional MC model, and applies Taylor approximation, to develop the Grey MC prediction model.

In order to develop the Grey MC prediction model, it has to transfer the Grey number. This transaction is named the whitening transformation of the Grey number. In our study, we can whiten this Grey number by following Equation (12): 


$$
\otimes v_{i}=\alpha_{i} L_{i}+\left(1-\alpha_{i}\right) U_{i} \quad i=1,2, \ldots, r
$$

In this equation, $\alpha_{i} \in[0,1]$ is the whitening coefficient. By combining this with Equation (11), the Grey Markov Chain model can be developed as follows:

$$
\widetilde{x}^{(0)}(t+1)=\hat{x}^{(0)}(t+1)+\sum_{i}^{r} a_{i}(t)\left[\alpha_{i} L_{i}+\left(1-\alpha_{i}\right) U_{i}\right]
$$

Based on Equation (13), if we can confirm vectors $\left[\alpha_{1}, \alpha_{2}, \ldots, \alpha_{r}\right]$, the Grey Markov Chain model can provide the prediction. Hence, the following section will apply Taylor approximation to confirm $\left[\alpha_{1}, \alpha_{2}, \ldots, \alpha_{r}\right]$ in the minimum convergence tolerance. The solution is provided as follows.

Step 1: Initialization

Set the number of iterations as K.

Set the initial whitening coefficient as:

$$
\alpha_{i}=\left[\alpha_{1}, \alpha_{2}, \ldots, \alpha_{r}\right]^{T}=[0.5,0.5, \ldots, 0.5]^{T}
$$

Set the objective function vector as:

$$
G=\left[x^{(0)}(1), x^{(0)}(2), \ldots, x^{(0)}(n)\right]^{T}
$$

Set the approximate function vector as:

$$
F^{(K)}=\left[\widetilde{x}^{(0)(K)}(1), \widetilde{x}^{(0)(K)}(2), \ldots, \widetilde{x}^{(0)(K)}(n)\right]^{T}
$$

Set approximate parameter as:

$$
\alpha^{(K)}=\left[\alpha_{1}^{(K)}, \alpha_{2}^{(K)}, \ldots, \alpha_{r}^{(K)}\right]^{T}
$$

Step 2: Calculate the approximate function vector $F^{(K)}$ by applying Taylor series expansion:

$$
\begin{gathered}
F^{(K+1)}=F^{(K)}+\sum_{i=1}^{r} F_{i}^{(K)}\left[\alpha_{i}^{(K+1)}-\alpha_{i}^{(K)}\right] \\
F_{1}^{(K)}=\frac{\partial F^{(K)}}{\partial \alpha_{1}^{(K)}} \approx \frac{F^{(K)}\left[\alpha_{1}^{(K)}+C_{1}^{(K)}\right]-F^{(K)} \alpha_{1}^{(K)}}{C_{1}^{(K)}} \\
F_{2}^{(K)}=\frac{\partial F^{(K)}}{\partial \alpha_{2}^{(K)}} \approx \frac{F^{(K)}\left[\alpha_{2}^{(K)}+C_{2}^{(K)}\right]-F^{(K)} \alpha_{2}^{(K)}}{C_{2}^{(K)}} \\
\vdots \\
F_{r}^{(K)}=\frac{\partial F^{(K)}}{\partial \alpha_{r}^{(K)}} \approx \frac{F^{(K)}\left[\alpha_{r}^{(K)}+C_{r}^{(K)}\right]-F^{(K)} \alpha_{r}^{(K)}}{C_{r}^{(K)}}
\end{gathered}
$$

In Equation (20), and:

$$
C_{1}^{(K)}=\frac{\alpha_{1}^{(K)}}{R_{1}}, C_{2}^{(K)}=\frac{\alpha_{2}^{(K)}}{R_{2}}, \ldots, C_{r}^{(k)}=\frac{\alpha_{r}^{(K)}}{R_{r}}
$$

where $R_{1}, R_{2}, \ldots, R_{r}$ is the step length coefficient, which is set as $R_{1}=R_{2}, \ldots, R_{r}=200$ in this study.

Step 3: Set the evaluation function as: 


$$
\begin{gathered}
Q^{(K)}=\left[F_{D}^{(K)}-\sum_{i=1}^{r} F_{i}^{(K)} \alpha_{i}\right]^{T}\left[F_{D}^{(K)}-\sum_{i=1}^{r} F_{i}^{(K)} \alpha_{i}\right] \text { where } F_{D}^{(K)}=G-F^{(K)} \\
\eta^{(K)}=\left[\begin{array}{c}
\eta_{1}^{(K)} \\
\eta_{2}^{(K)} \\
\vdots \\
\eta_{r}^{(K)}
\end{array}\right]=\left[\begin{array}{c}
\alpha_{1}^{(K+1)}-\alpha_{1}^{(K)} \\
\alpha_{2}^{(K+1)}-\alpha_{2}^{(K)} \\
\vdots \\
\alpha_{r}^{(K+1)}-\alpha_{r}^{(K)}
\end{array}\right]
\end{gathered}
$$

If we assume a tolerable error $\varepsilon$, at the point which the evaluation function iteration terminates and enters step 4.

Step 4: Iterate the approximate parameter:

$$
\operatorname{Min}\left(Q^{(K)}\right) \rightarrow 0
$$

Let:

$$
\frac{\partial Q^{(K)}}{\partial \eta_{1}^{(K)}}=0, \frac{\partial Q^{(K)}}{\partial \eta_{2}^{(K)}}=0, \ldots, \frac{\partial Q^{(K)}}{\partial \eta_{r}^{(K)}}=0
$$

It can achieve Equation (25).

$$
\alpha^{(K+1)}=\alpha^{(K)}+\left[A^{(K) T} A^{(K)}\right]^{-1} A^{(K) T} F_{D}^{(K)}, \text { and } A^{(K)}=\left[F_{1}^{(K)}, F_{2}^{(K)}, \ldots, F_{r}^{(K)}\right]
$$

Lastly, we need to increase the number of iterations $(K=K+1)$, and repeat the step 2. When $Q^{(K)} \leq \varepsilon$, the predicting value of the improved Grey MC model is:

$$
F^{(K)}=\left[\widetilde{x}^{(0)(K)}(1), \widetilde{x}^{(0)(K)}(2), \ldots, \widetilde{x}^{(0)(K)}(n)\right]^{T}
$$

The T-MCGM $(1,1)$ model we developed is unique in three main aspects. First, it has the advantage of creating the prediction without requiring large databases or sample sizes. Second, the Markov Chain model requires the object to be stationary, while the object of T-MCGM $(1,1)$ can be non-stationary. Third, T-MCGM $(1,1)$ is able to revise the volatility and noise in the prediction. Thus, it has a better performance than ARMA and BP in terms of predicated accuracy. To better verify the effectiveness of T-MCGM $(1,1)$, the following section applies it with empirical data, and compares the results with other prediction models.

\subsection{Data}

We used the database of China Health Statistic Yearbook, and obtain the medical services demand of diabetes DD), heart disease (HD) and cerebrovascular disease (CD). These three types of disease are very common in the aging population in China, and they have features that include high incidence rate, multiple causes, and other random factors. Therefore, these diseases can be used as an ideal case to test the improved Grey MC model. Hospitalizing rate within two-weeks is selected as the indicators. The data of hospitalizing rate within two-weeks from 2006 to 2015 are listed in Table 1.

Table 1. Original data from 2006 to 2015.

\begin{tabular}{ccccccccccc}
\hline Year & $\mathbf{2 0 0 6}$ & $\mathbf{2 0 0 7}$ & $\mathbf{2 0 0 8}$ & $\mathbf{2 0 0 9}$ & $\mathbf{2 0 1 0}$ & $\mathbf{2 0 1 1}$ & $\mathbf{2 0 1 2}$ & $\mathbf{2 0 1 3}$ & $\mathbf{2 0 1 4}$ & $\mathbf{2 0 1 5}$ \\
\hline DD & 2.3 & 3 & 3.3 & 7.6 & 8.2 & 9.3 & 10.7 & 11.4 & 10.5 & 12.5 \\
HD & 12.2 & 11.4 & 10.2 & 11.9 & 13.5 & 14.7 & 16.1 & 17.5 & 18.3 & 19.9 \\
CD & 2.7 & 6.7 & 3.6 & 5.1 & 6.9 & 7.5 & 8.9 & 10.7 & 11.6 & 12.3 \\
\hline
\end{tabular}




\subsection{Procedure}

Based on Table 1, we test the predicting accuracy of the traditional GM $(1,1)$ model. By importing the data in Table 1 into GM $(1,1)$, we can achieve the prediction and errors. In Figures 1-3, the blue line represents the original data in Table 1 . The red line represents the prediction which is calculated by the traditional GM $(1,1)$ model. It can be seen that the two periods include 2008 to 2013 and 2013 to 2015 have significant errors in Figure 1. It can be seen that two periods including 2007 to 2012 and 2013 to 2015 have significant errors in Figure 2. It can also be seen that one period of 2008 to 2011 has significant errors in Figure 3. Due to the errors, the GM $(1,1)$ model has a weak performance in predicting the medical services demands. Therefore, we purposely apply the same data, and test them using the improved MC model by Taylor Approximation.

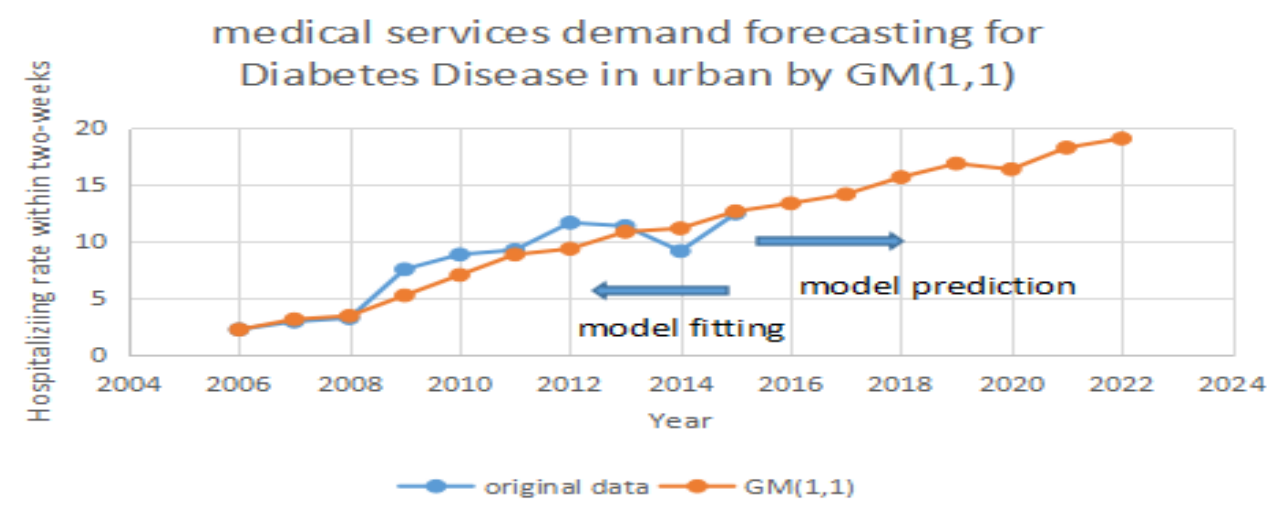

Figure 1. Forecasting diabetes in urban areas by GM $(1,1)$.

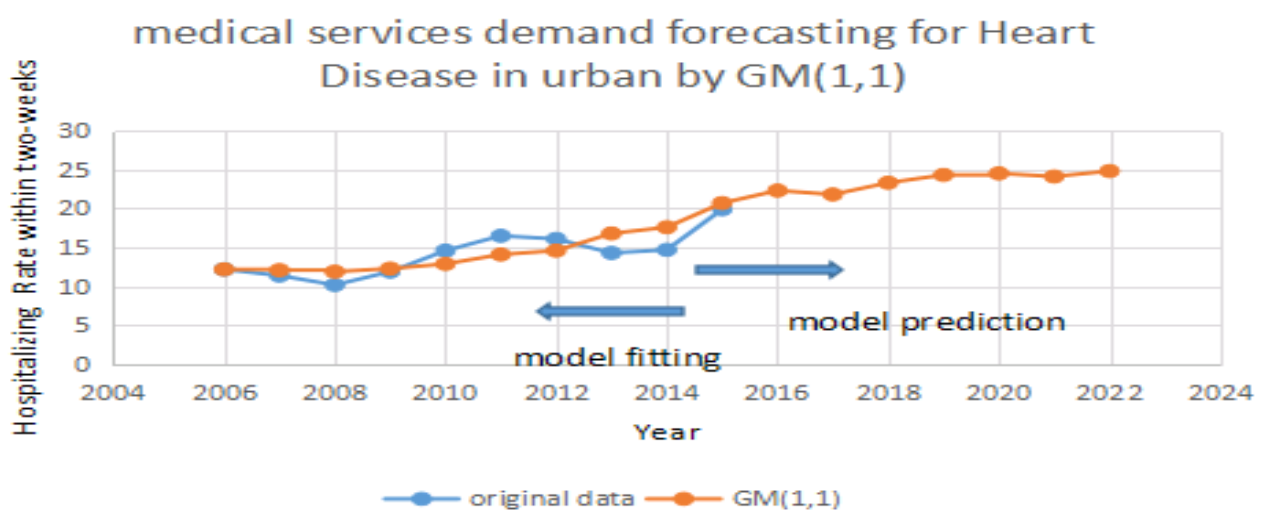

Figure 2. Forecasting for heart disease in urban areas by GM $(1,1)$.

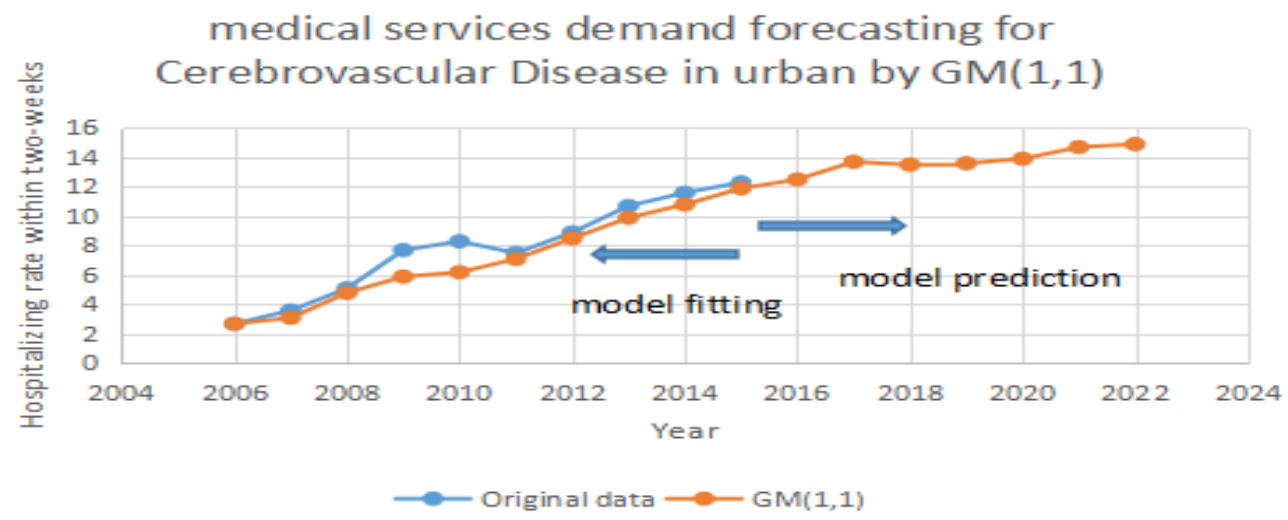

Figure 3. Forecasting cerebrovascular disease in urban areas by $\mathrm{GM}(1,1)$. 
After the model completes the iterations, it can achieve the optimization of the vectors $\left[\alpha_{1}, \alpha_{2}, \ldots, \alpha_{r}\right]$. By applying Equation (13), it can calculate the prediction of the T-MCGM $(1,1)$ model. In Figure 4, the blue line stands for the original date, the red line represents the prediction of the T-MCGM $(1,1)$ model.

Based on Figures 4-6, the T-MCGM $(1,1)$ model has performed a better prediction than the traditional GM $(1,1)$ model. Especially for the periods with significant errors by GM $(1,1)$, the prediction of T-MCGM $(1,1)$ is more accurate, and has smaller errors than the traditional GM $(1,1)$ model. Specifically, the prediction precision in Figure 2 by GM $(1,1)$ (MAPE) is $10.3 \%$, and the prediction precision in Figure 5 by T-MCGM $(1,1)$ is $6.23 \%$. From the perspective of long-term prediction, the improvement of the prediction accuracy is acceptable. Moreover, the T-MCGM $(1,1)$ model is based on GM $(1,1)$, and the result by T-MCGM $(1,1)$ is obtained by revising the forecasting result. Therefore, improvement of the prediction accuracy is not significant.

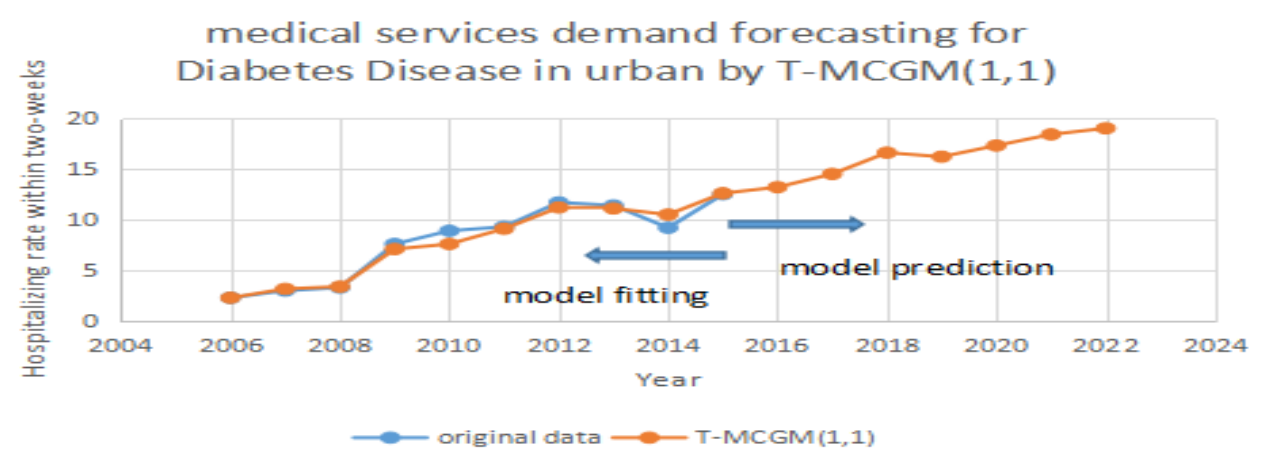

Figure 4. Forecasting diabetes in urban areas by T-MCGM $(1,1)$.

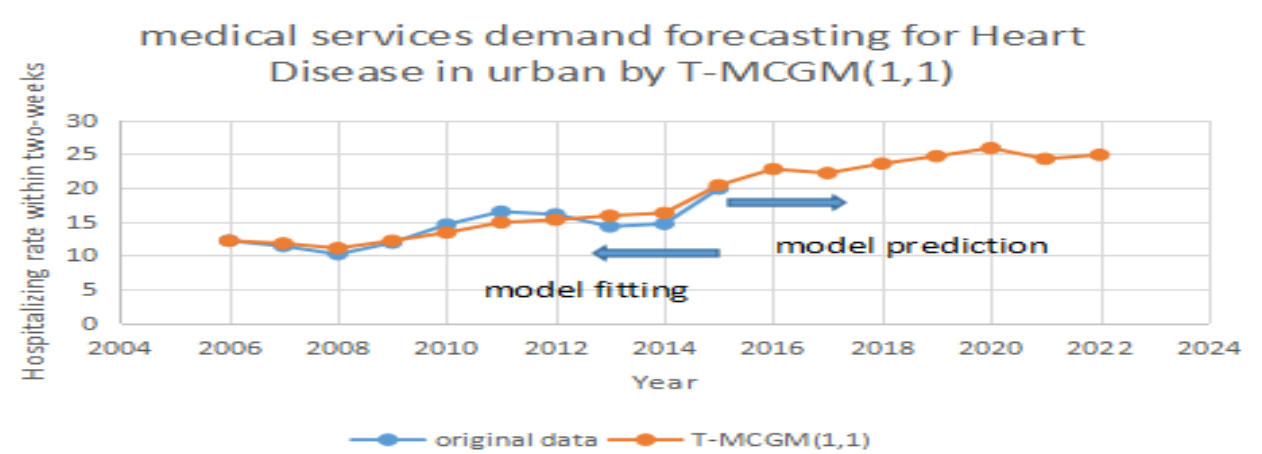

Figure 5. Forecasting heart disease in urban areas by T-MCGM $(1,1)$.

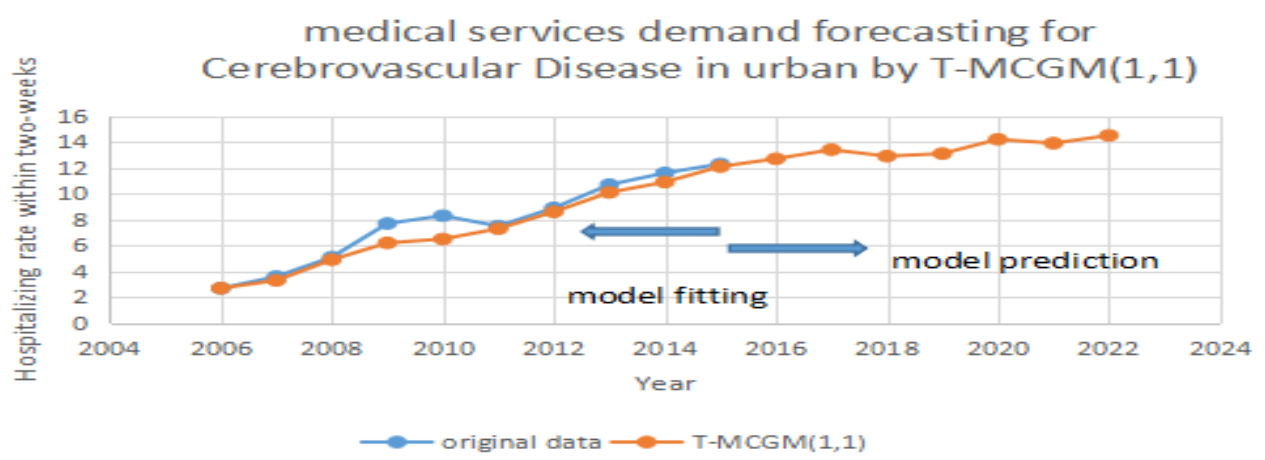

Figure 6. Forecasting cerebrovascular disease in urban areas by T-MCGM $(1,1)$. 


\section{Results}

In order to test the feasibility of T-MCGM $(1,1)$ model, our study selects other three models which include auto regressive moving average (ARMA), back propagation neural network (BP), and GM $(1,1)$ to compare with T-MCGM $(1,1)$. The test focuses on two criteria, including absolute mean percentage error (MAPE) and root mean square error (RMSE), to compare the four models' performance in these criteria. The result of the comparison is presented in Figure 7 and Table 2.

Table 2. The prediction precision by four various models.

\begin{tabular}{ccccccc}
\hline \multirow{2}{*}{ Model } & \multicolumn{2}{c}{ Diabetes Disease } & \multicolumn{2}{c}{ Heart Disease } & \multicolumn{2}{c}{ Cerebrovascular } \\
\cline { 2 - 7 } & MAPE & RMSE & MAPE & RMSE & MAPE & RMSE \\
\hline ARMA & $11.68 \%$ & 0.5427 & $13.53 \%$ & 0.7011 & $11.32 \%$ & 0.4936 \\
BP & $12.77 \%$ & 0.6481 & $12.35 \%$ & 0.5673 & $11.21 \%$ & 0.4922 \\
GM $(1,1)$ & $11.54 \%$ & 0.4284 & $10.30 \%$ & 0.5453 & $9.59 \%$ & 0.3254 \\
T-MCGM $(1,1)$ & $5.66 \%$ & 0.2016 & $6.23 \%$ & 0.3333 & $6.31 \%$ & 0.2577 \\
\hline
\end{tabular}

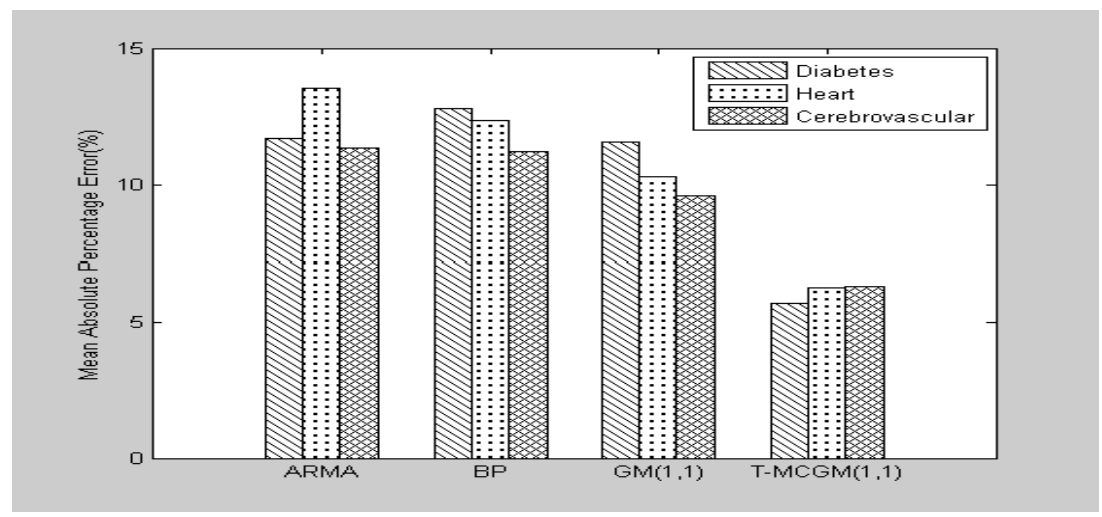

Figure 7. The comparison of fitting results by four models.

As shown in Figure 7 and Table 2, T-MCGM $(1,1)$ has the highest imitation with the original data. Comparing with the traditional GM $(1,1)$ model, T-MCGM $(1,1)$ has reduced $50.95 \%$ in MAPE for diabetes. Comparing with BP and ARMA, T-MCGM $(1,1)$ has decreased $55.68 \%$ and $51.54 \%$ in MAPE for diabetes, respectively. These results are able to prove the feasibility of T-MCGM $(1,1)$ in our study, which has a better prediction on medical services demands than the other three models.

Table 3 shows the forecasting value from 2016 to 2022 for medical services demand by T-MCGM (1,1). For diabetes, there is a significant growth from 2017 to 2018, and from 2018 to 2022, it presents a relatively stable growth. For heart disease, there are two shocks from 2016 to 2017 and from 2020 to 2021. For cerebrovascular disease, there are two periods in slight decline from 2016 to 2017, and from 2021 to 2022.

Table 3. The prediction results for three types of disease by T-MCGM $(1,1)(\% 0)$.

\begin{tabular}{cccccccc}
\hline Year & $\mathbf{2 0 1 6}$ & $\mathbf{2 0 1 7}$ & $\mathbf{2 0 1 8}$ & $\mathbf{2 0 1 9}$ & $\mathbf{2 0 2 0}$ & $\mathbf{2 0 2 1}$ & $\mathbf{2 0 2 2}$ \\
\hline DD & 13.2 & 14.5 & 16.6 & 16.2 & 17.3 & 18.4 & 19 \\
HD & 22.8 & 22.2 & 23.6 & 24.7 & 25.9 & 24.3 & 24.9 \\
CD & 12.7 & 13.4 & 12.9 & 13.1 & 14.2 & 13.9 & 14.5 \\
\hline
\end{tabular}

\section{Discussion}

By applying the T-MCGM $(1,1)$ model in urban areas of China, we have noticed that there will be an increase of the demand of medical services from 2016 to 2022. Regarding the types of disease discussed above, some suggestions are provided to be considered in further medical resource allocation. 
The demand for medical services in diabetes and cerebrovascular disease has faster increasing rates than heart disease. This can be explained as individuals older than 65 are the main patients of the first two. Different diseases require different medical resources. For example, the patients with chronic disease need plenty of drug resources and nursing staff, while patients with serious acute disease need surgical treatment. Therefore, medical service prediction should consider the different types of disease.

There is a larger fitting error in the demand fitting curve in 2010-2011. This may be caused by the New Medical Reform Policy which has been practiced since 2009. The scope of urban medical insurance is expanding and the proportion of reimbursement is increasing. The demand of medical services for chronic disease is stimulated. The prediction models based on GM $(1,1)$ are weak, as without considering policy-driving factors, the demand increase of the prediction for medical services presents the hysteresis characteristics.

The individual living standards and individual health awareness have increased in many developing countries in recent years. Therefore, the T-MCGM $(1,1)$ model for medical services prediction can be applied to these countries for releasing their limited medical resources, such as India and Brazil. It is especially able to contribute a more accurate medical demand prediction in these countries, and provide adequate resources for medical service provision.

The prediction of the demand of medical services for specific diseases has practical implications for the medical and health authorities, hospitals, medical colleges, and drug manufacturers. Different diseases require different medical resources. For example, the patients with chronic disease need plenty of drug resources and nursing staff, while patients with serious acute disease need surgical treatment and beds in hospitals. In this paper, the forecasting results from 2016 to 2022 can promote health service organizations to allocate limited medical resources, including doctors, nurses, pharmacists, and other human resources and drug resources. However, due to the limited access to the administration data on medical resources allocation, the practice of optimizing limited medical resources has not been discussed from a technical perspective in this paper. This aspect needs to be simulated and studied in further research.

Generally, T-MCGM $(1,1)$ overcomes the shortage of omitting the driving factors in the GM $(1,1)$ model, and also weakens the volatility and noisiness of data by combining the Markov chain. Regarding this advantage, T-MCGM $(1,1)$ has presented good adaptability for poor data and small samples. With using the same group of historical data of medical service demands, the model has a better prediction than the ARMA and BP models. In terms of the volatility and noisy of databases in medical service demands, the T-MCGM $(1,1)$ model is more suitable for predicting the demands of patients.

However, the development of T-MCGM $(1,1)$ requires combining the three different models, which are based on a large amount of calculation and time consumption. Thus, it is not easy to carry out for solving urgent predictions of medical service demands and allocations of health resources in reality. Further research includes considering the prediction on rural areas in China, and focusing on comparing the difference between urban areas and rural areas in medical services demand. In this regard, the further research aims to contribute more allocating schemes for urban and rural areas.

\section{Conclusions}

The T-MCGM $(1,1)$ model represents a feasible and efficient method for predicting the trend of annual medical services demand for various diseases in China. The prediction results can be used by the national health administration to allocate limited medical resources, and for develop hospitals and other health organizations to develop proactive service plans.

Acknowledgments: We would like to thank Research Project of Education Department of Fujian Province (grant no. JAS150290), and Soft Science Research Project of Fujian Province (grant no. 2017R0048) for funding to this research project. We are also grateful to the editor and anonymous reviewers for their helpful comments and suggestions.

Author Contributions: Jinli Duan: conception and design, analysis and interpretation of data; writing and critically revising the manuscript. Feng Jiao: writing and critically revising the manuscript. Qishan Zhang: conception and design, analysis and interpretation of data. Zhibin Lin: writing and critically revising the manuscript. 
Conflicts of Interest: The authors declare no conflict of interest.

\section{References}

1. Deb, P.; Trivedi, P.K. Demand for medical care by the elderly: A finite mixture approach. J. Appl. Econ. 1997, 12, 313-336.

2. Parker, M.G.; Thorslund, M. Health trends in the elderly population: Getting better and getting worse. Gerontologist 2007, 47, 150-158. [CrossRef] [PubMed]

3. Lehnert, T.; Heider, D.; Leicht, H.; Heinrich, S.; Corrieri, S.; Luppa, M.; König, H.H. Review: Health care utilization and costs of elderly persons with multiple chronic conditions. Med. Care Res. Rev. 2011, 68, 387-420. [PubMed]

4. Umberson, D.; Montez, J.K. Social relationships and health a flashpoint for health policy. J. Health Soc. Behav. 2010, 51 (Suppl. 1), S54-S66. [CrossRef] [PubMed]

5. Grossman, M. On the concept of health capital and the demand for health. J. Political Econ. 1972, 80, $223-255$. [CrossRef]

6. Hupert, N.; Wattson, D.; Cuomo, J.; Benson, S. Anticipating Demand for Emergency Health Services due to Medication-related Adverse Events after Rapid Mass Prophylaxis Campaigns. Acad. Emerg. Med. 2007, 14, 268-274. [PubMed]

7. McCarthy, M.L.; Zeger, S.L.; Ding, R.; Aronsky, D.; Hoot, N.R.; Kelen, G.D. The challenge of predicting demand for emergency department services. Acad. Emerg. Med. 2008, 15, 337-346. [CrossRef] [PubMed]

8. Lowthian, J.A.; Curtis, A.J.; Jolley, D.J.; Stoelwinder, J.U.; McNeil, J.J.; Cameron, P.A. Demand at the emergency department front door: 10-year trends in presentations. Med. J. Aust. 2012, 196, 128-132. [PubMed]

9. Hagihara, A.; Hasegawa, M.; Hinohara, Y.; Abe, T.; Motoi, M. The aging population and future demand for emergency ambulances in Japan. Intern. Emerg. Med. 2013, 8, 431-437. [CrossRef] [PubMed]

10. Zhang, Y.; Liang, L.; Liu, E.; Chen, C.; Atkins, D. Patient choice analysis and demand prediction for a health care diagnostics company. Eur. J. Oper. Res. 2016, 251, 198-205.

11. Liu, J.X.; Goryakin, Y.; Maeda, A.; Bruckner, T.; Scheffler, R. Global Health Workforce Labor Market Projections for 2030. Hum. Resour. Health 2017, 15, 1-12. [CrossRef] [PubMed]

12. Jäger, R.; Berg, N.; Hoffmann, W. Estimating future dental services' demand and supply: A model for Northern Germany. Community Dent. Oral Epidemiol. 2016, 44, 169-179. [CrossRef] [PubMed]

13. Gudleski, G.D.; Satchidanand, N.; Dunlap, L.J. Predictors of medical and mental health care use in patients with irritable bowel syndrome in the United States. Behav. Res. Ther. 2017, 88, 65-75. [CrossRef] [PubMed]

14. Jalalpoura, M.; Gelb, Y.; Levincd, S. Forecasting demand for health services: Development of a publicly available toolbox. Oper. Res. Health Care 2015, 5, 1-9. [CrossRef]

15. Veser, A.; Sieber, F.; Groß, S.; Prückner, S. The demographic impact on the demand for emergency medical services in the urban and rural regions of Bavaria, 2012-2032. J. Public Health 2015, 23, 181-188. [CrossRef] [PubMed]

16. Zhu, Y.; Xia, J.L.; Wang, J. Comparison of predictive effect between the single auto regressive integrated moving average (ARIMA) model and the ARIMA-generalized regression neural network (GRNN) combination model on the incidence of scarlet fever. Chin. J. Epidemiol. 2009, 30, 964-968.

17. Yu, S.J.; Zhu, C.H.; Li, J. Cardiovascular Hospital Medical Service Demand Estimation. Chin. Hosp. Archit. Equip. 2014, 15, 99-103.

18. Li, Y.Z.; Zhang, T.; Wan, L.Z. Estimation of Demand Volume for Basic Public Health Services Based on Age-gender Population Structure. Chin. Gen. Pract. 2016, 19, 400-403.

19. Landry, M.D.; Hack, L.M.; Coulson, E.; Freburger, J.; Johnson, M.P.; Katz, R.; Kerwin, J.; Smith, M.H.; Venskus, D.G.; Sinnott, P.L.; et al. Workforce Projections 2010-2020: Annual Supply and Demand Forecasting Models for Physical Therapists Across the United States. Phys. Ther. 2016, 96, 71-80. [CrossRef] [PubMed]

20. Yu, W.; Li, M.; Ge, Y.; Li, L.; Zhang, Y.; Liu, Y.; Zhang, L. Transformation of Potential Medical Demand in China: A System Dynamics Simulation Model. J. Biomed. Inform. 2015, 57, 399-414. [CrossRef] [PubMed]

21. Cardoso, T.; Oliveira, M.D.; Barbosa-Póvoa, A. Modeling the demand for long-term care services under uncertain information. Health Care Manag. Sci. 2012, 15, 385-412. [CrossRef] [PubMed] 
22. Akita, T.; Tanaka, J.; Ohisa, M. Predicting future blood supply and demand in Japan with a Markov model: Application to the sex- and age-specific probability of blood donation. Transfusion 2016, 56, 2750-2759. [CrossRef] [PubMed]

23. Kumar, U.; Jain, V.K. Time Series models (Grey-markov, Grey Model with rolling mechanism and Singular Spectrum analysis) to Forecast Energy Consumption in India. Energy 2010, 35, 1709-1716. [CrossRef]

24. Chen, L.H.; Guo, T.S. Forecasting Financial crises for an enterprise by using the Grey Markov forecasting model. Qual. Quant. 2011, 45, 911-922. [CrossRef]

25. Lin, L.C.; Wu, S.Y. Analyzing Taiwan IC Assembly Industry by Grey-Markov Forecasting Model. Math. Probl. Eng. 2013, 2013, 1024-1030. [CrossRef]

26. Kordnoori, S.; Mostafaei, H.; Kordnoon, S. The Application of Fourier Residual Grey Verhulst and Grey Markov Model in Analyzing the Global ICT Development. Hyperion Econ. J. 2014, 2, 50-60.

27. Edem, I.E.; Oke, S.A.; Adebiyi, K.A. A modified grey-Markov Fire Accident Model Based on Information Turbulence Indices and Restricted Residuals. Int. J. Manag. Sci. Eng. Manag. 2016, 11, 231-242. [CrossRef]

28. Peng, R. Analysis of Senior Population Nursing Needs Based on Markov Model. Stat. Inf. Forum 2009, 24, 77-80.

29. Huang, F.; Wu, C. A Study of Long-Term-Care Demand of the Elderly in China: Based on Multistatus Transition Model. Econ. Res. J. 2012, 47, 119-130.

30. Hu, H.; Li, Y.; Zhang, L. Estimation and Prediction of Demand of Chinese Elederly Long-term Care Services. Chin. J. Popul. Sci. 2015, 13, 79-89.

31. Argiento, R.; Guglielmi, A.; Lanzarone, E.; Nawajah, I. A Bayesian framework for describing and predicting the stochastic demand of home care patients. Flex. Serv. Manuf. J. 2016, 28, 254-279. [CrossRef]

32. Deng, J. Introduction to grey system theory. J. Grey Syst. 1989, 1, 1-24.

33. Lin, Y.; Liu, S.F. A historical introduction to grey systems theory. In Proceedings of the 2004 IEEE International Conference on Systems, Man, and Cybernetics, Hague, The Netherlands, 10-13 October 2004; Volume 3, pp. 2403-2408.

34. Deng, J. Control problems of grey system. Syst. Control Lett. 1982, 1, 288-294.

35. Deng, J. Extent Information Cover in Grey System Theory. J. Grey Syst. 1995, 7, 13-25.

36. Chen, F.Y.; Zhang, L.Y.; Yang, W.X. Analysis and Prediction of The Supply and Demand of Bed Resources in Tianjin City. Chin. J. Health Stat. 2012, 29, 404-405.

37. Xiang, H.L.; Guo, S.X.; Tang, J.X.; Cao, L.; Liu, X.P.; Liu, J.M.; Guo, H.; Liu, H.T.; Li, S.G. Based on multi-factors grey model to predict demand for community health workforce. Chin. J. Health Stat. 2015, 32, 493-495.

38. Bao, C.Z.; Mayila, M.; Ye, Z.H.; Wang, J.B.; Jin, M.J.; He, W.J.; Chen, K. Forecasting and Analyzing the Disease Burden of Aged Population in China, Based on the 2010 Global Burden of Disease Study. Int. J. Environ. Res. Public Health 2015, 12, 7172-7184. [CrossRef] [PubMed]

39. Wang, Y.X.; Du, Q.Y.; Ren, F.; Liang, S.; Lin, D.; Tian, Q.; Chen, Y.; Li, J. Spatio-Temporal Variation and Prediction of Ischemic Heart Disease Hospitalizations in Shenzhen, China. Int. J. Environ. Res. Public Health 2014, 11, 4799-4824. [CrossRef] [PubMed]

40. Li, G.-D.; Yamaguchi, D; Nagai, M. A GM (1,1)-Markov chain combined model with an application to predict the number of Chinese international airlines. Technol. Forecast. Soc. Chang. 2007, 74, 1465-1481.

(C) 2017 by the authors. Licensee MDPI, Basel, Switzerland. This article is an open access article distributed under the terms and conditions of the Creative Commons Attribution (CC BY) license (http://creativecommons.org/licenses/by/4.0/). 